\title{
Chromosomal and Molecular Abnormalities in a Group of Brazilian Infertile Men with Severe Oligozoospermia or Non-Obstructive Azoospermia Attending an Infertility Service
}

\author{
Fernanda A. Mafra, Denise M. Christofolini, Bianca Bianco, Marcello M. Gava, Sidney Glina, \\ Sintia I. N. Belangero, Caio P. Barbosa
}

Division of Pathological Gynecology and Human Reproduction (FAM, DMC, BB, MMG, SINB, $C P B)$, Department of Gynecology and Obstetrics and Division of Urology (MMG, SG), Department of Surgery, ABC School of Medicine, Santo Andre, Sao Paulo, Brazil

\begin{abstract}
Purpose: To determine the frequency of genetic alterations in a population of Brazilian infertile men with severe oligozoospermia or non-obstructive azoospermia.

Materials and Methods: Retrospective study of a group of 143 infertile men with severe oligozoospermia or non-obstructive azoospermia from the Andrology Outpatient Clinic of the Human Reproduction Service at the ABC School of Medicine. Of these patients, 100 had severe oligozoospermia, and 43 non-obstructive azoospermia. All patients underwent a genetic study which included karyotype analysis and Y-microdeletion investigation.

Results: Genetic abnormalities were found in $18.8 \%$ of the studied patients. Chromosomal abnormalities were found in $6.2 \%$ of the patients, being more prevalent in the azoospermia group (11.6\%) than in the oligozoospermia group (4\%). Chromosomal variants were found in $8.3 \%$, and Y-chromosome microdeletions in $4.2 \%$ of patients.

Conclusion: The high frequency of genetic alterations (18.8\%) in our series justified performing a genetic investigation in a population with idiopathic infertility, as results may help determine the prognosis, as well as the choice of an assisted reproduction technique. Moreover, a genetic investigation could minimize the risk of transmitting genetic abnormalities to future generations such as genetic male infertility, mental retardation, genital ambiguity and/or birth defects.
\end{abstract}

Key words: male infertility; chromosome abnormalities; Y chromosome; microdeletions; oligozoospermia; azoospermia Int Braz J Urol. 2011; 37: 244-51

\section{INTRODUCTION}

Infertility is a very common health problem, affecting approximately $15-20 \%$ of couples who attempt pregnancy. In almost $50 \%$ of infertile couples, the problem is related to the male. About $15 \%$ of infertile men may carry a genetic abnormality, including chromosomal aberrations and single-gene mutations $(1,2)$.
The frequency of chromosomal aberrations in subfertile males is estimated to be $2-3 \%$, and in infertile patients with sperm counts below $10 \times 106$ spermatozoa/mL may reach $5-7 \%$; among patients with azoospermia, the percentage of individuals with cytogenetic abnormalities increases by $10-15 \%$ $(1,2)$.

Among the genetic abnormalities found in infertile men, those involving chromosome anomalies 
amount to about $8 \%$, the most frequent one being the 47,XXY karyotype that characterizes the Klinefelter Syndrome (1). In comparison, it is worth mentioning that, in the general population, Robertsonian and reciprocal translocations are present in $1 / 1000$ and $0.9 / 1000$ newborns, respectively, therefore the incidence in the infertile male population is nine and seven times higher, respectively (1).

Microdeletions of the $\mathrm{Y}$ chromosome are also important causes of male infertility, and there are studies showing that deletions of azoospermia factor (AZF) loci on the long arm of the Y chromosome are associated with either reduced sperm count (oligozoospermia) or complete absence of spermatozoa (azoospermia). Microdeletions in these regions are a cause of severe testiculopathy and are present in $2 \%$ of infertile men (3). In selected males affected by severe oligozoospermia or non-obstructive azoospermia, the frequency of $\mathrm{Y}$ microdeletions can be increased to as much as $15-20 \%$ (4).

The aim of this study was to determine the frequency of chromosomal abnormalities and Ychromosome microdeletions in a group of Brazilian infertile men attending an infertility service.

\section{MATERIALS AND METHODS}

This study made a retrospective assessment of the data of 143 infertile men (age range 25 to 52 years, mean: $36.6 \pm 5.6$ years) with non-obstructive sperm disorders, seen between June 2006 and September 2008 at the Andrology Outpatient Clinic of the ABC School of Medicine, Santo André/SP, Brazil. All patients included presented one or more years of primary infertility and had at least two semen analyses showing either sperm counts under 5 million/mL or azoospermia, with no suspicion of an obstructive cause (medical history, physical examination and complementary tests). All participants gave informed consent, according to the protocol approved by the local Ethics Committee (FMABC No. 237/2008).

Routine clinical and laboratory tests were performed on all patients, including semen analysis, karyotype analysis and investigation of $\mathrm{Y}$ chromosome microdeletions. Semen analysis was performed according to the guidelines of the World Health Organization (5).

Karyotype analysis was performed using G-banding, and at least 20 peripheral blood metaphases were analyzed for each patient. The number of analyzed metaphases was increased to 100 whenever necessary. The karyotype results were described according to the International System for Human Cytogenetic Nomenclature (6).

Fluorescent in situ hybridization (FISH) with X- and Y-chromosome centromeric probes and a sex-determining region $\mathrm{Y}(S R Y)$ probe (Cytocell®, Cambridge, UK) was performed according to the protocol of Pinkel et al. (1988) (7). One hundred nuclei were analyzed with the ISIS Software (MetaSystems $\mathrm{GmbH}$, Altlussheim, Germany) and the results were described according to the International System for Human Cytogenetic Nomenclature (6).

For the molecular study, genomic DNA was extracted from lymphocytes, using the Illustra ${ }^{\mathrm{TM}}$ Blood GenomicPrep Mini Spin Kit (GE Healthcare Life Sciences, Buckinghamshire, UK), according to the manufacturer's instructions. Multiplex PCR was performed for $S R Y$ (Yp), $D B Y$ (AZFa), $R B M Y$ (AZFb), DAZ (AZFc), AMELX (X-chromosome control) and AMELY (Y-chromosome control), according to Umeno et al. (2006) (8), with temperature modifications. A gene was considered absent only after three amplification failures, in the presence of amplification of the internal control $(A M E L X)$ and positive control.

\section{RESULTS}

Genetic abnormalities were found in $18.8 \%$ of all infertile men studied, as shown in Table- 1 . The frequency of chromosomal abnormalities was $6.2 \%$ (9/143), and is shown in Table-2. Considering only the patients with severe oligozoospermia, the frequency of chromosomal abnormalities was 4.0\% (4/100), while in those with azoospermia it was $11.6 \%(5 / 43)$. Chromosomal variants or polymorphisms were found in $8.3 \%$ of all cases (Table-3).

Y-chromosome microdeletions were found in $4.2 \%(6 / 143)$ of all the infertile men studied. In a separate analysis, we found $3.0 \%(3 / 100)$ in the 
Table 1 - Genetic aberrations (chromosomal abnormalities, variants and Y microdeletions) in infertile men with azoospermia or severe oligozoospermia.

\begin{tabular}{lccr}
\hline \multirow{1}{*}{ Genetic Abnormalities } & \multicolumn{2}{c}{ Sperm Disorder } \\
& Azoospermia & Oligozoospermia & Total \\
& $\mathrm{N}-\%$ & $\mathrm{~N}-\%$ & $\mathrm{~N}-\%$ \\
\hline Chromosomal abnormalities & $5 / 43-11.6$ & $4 / 100-4.0$ & $9 / 143-6.2$ \\
Chromosomal variants & $3 / 43-6.9$ & $9 / 100-9.0$ & $12 / 143-8.4$ \\
Y-chromosome microdeletions & $3 / 43-6.9$ & $3 / 100-3.0$ & $6 / 143-4.4$ \\
Total & $11 / 43-25.4 \%$ & $16 / 100-16.0$ & $27 / 143-18.8$ \\
\hline
\end{tabular}

oligozoospermia group and $6.9 \%(3 / 43)$ in the azoospermia group. Of these six patients, three carried microdeletions of the AZFc region and presented severe oligozoospermia, two carried microdeletions of the $\mathrm{AZFb}+\mathrm{c}$ regions and presented non-obstructive azoospermia, and one had a deletion of AZFa and presented non-obstructive azoospermia.

\section{COMMENTS}

Genetic causes, such as chromosomal aberrations, single-gene mutations and Y-chromosome microdeletions, are found in a large proportion of infertile couples: they are present in about $15 \%$ of infertile men and $10 \%$ of infertile women (1).

Table 2 - Autosomal and sex chromosomal abnormalities in a sample of infertile men with azoospermia or severe oligozoospermia.

\begin{tabular}{lcc}
\hline $\begin{array}{c}\text { Chromosomal } \\
\text { Abnormalities }\end{array}$ & \multicolumn{2}{c}{ Sperm Disorder } \\
& Azoospermia & Oligozoospermia \\
\hline XXY & 4 & - \\
XXY/XY & - & 1 \\
XX & 1 & - \\
$\mathrm{t}(6 ; 12)$ & - & 1 \\
$\mathrm{t}(13,14)$ & - & 1 \\
Add $(9)(\mathrm{p} 34)$ & - & 1 \\
Total & 5 & 4 \\
\hline$t=$ chromosomal translocation; Add $=$ additional chromosome \\
material.
\end{tabular}

In a previous study, Pina-Neto et al. (2006) (9) found that $9.6 \%(16 / 165)$ of the patients had karyotype abnormalities. In agreement with that study, we found a $6.2 \%(9 / 143)$ frequency of chromosomal abnormalities, and their prevalence was higher in the patients with azoospermia (11.6\%) than in those with oligozoospermia (4.0\%). In the group of azoospermic patients, sex chromosome abnormalities such as $47, \mathrm{XXY}, 46, \mathrm{XX} / 47, \mathrm{XXY}$ and $46, \mathrm{XX}$ were predominant. We found $1 \%$ of patients with Klinefelter syndrome among those with severe oligozoospermia, and $9 \%$ among those with azoospermia.

The 46,XX sex differentiation disorder in males represents the most common condition in which testicular development occurs in the absence of a Y chromosome. This disorder occurs at a frequency of 1/25.000 newborns (1). Phenotypically the adults are similar to patients with Klinefelter syndrome, with normal male external genitalia, microrchidia and sterility. Molecular analyses have shown that in approximately two thirds of these cases there are Ychromosome sequences present in the genome, including the SRY gene (10). In our study population, there was one patient who presented a 46,XX karyotype. FISH using a $S R Y$ probe confirmed the presence of the SRY gene on the short arm of one X chromosome. This is a common finding, resulting from an abnormal crossing-over of pseudoautosomal regions of the $\mathrm{X}$ and $\mathrm{Y}$ chromosomes. This abnormal crossing-over can result in a 46,XX male $(S R Y+)$ and/or a $46, \mathrm{XY}$ female (SRY-).

On the other hand, in the group of oligozoospermic patients, structural chromosomal abnormalities, such as balanced translocations, Robertsonian 
Chromosomal and Molecular Abnormalities in Infertile Man

Table 3 - Normal chromosomal variants (heteromorphisms) associated to sperm disorders in a sample of infertile men.

\begin{tabular}{ccc}
\hline Karyotype & N of Patients & Sperm Disorder \\
\hline $46, \mathrm{XY}, 9 \mathrm{ph}$ & 5 & Severe oligozoospermia \\
$46, \mathrm{XY}, 9 \mathrm{qh}+$ & 1 & Severe oligozoospermia \\
$46, \mathrm{XY}, 9 \mathrm{ph}, 9 \mathrm{qh}+$ & 1 & Severe oligozoospermia \\
$46, \mathrm{XY}, 14 \mathrm{ps}^{+}$ & 1 & Non-obstructive azoospermia \\
$46, \mathrm{XY}, 15 \mathrm{ps}^{+}$ & 1 & Severe oligozoospermia \\
$46, \mathrm{XY}, 21 \mathrm{ps}^{+}$ & 2 & Non-obstructive azoospermia \\
$46, \mathrm{XY}, 22 \mathrm{ps}^{+}$ & 1 & Severe oligozoospermia \\
\hline
\end{tabular}

translocations and additional chromosomal material, were more common, as shown in Table-2. Balanced forms of reciprocal translocations usually do not have any phenotypic effect on the carriers, except that they may show variable sperm counts, ranging from normal to low (oligozoospermia) or even total absence of spermatozoa (azoospermia) in the ejaculate (11). However, the offspring of reciprocal translocation carriers, such as the patient with a translocation between chromosomes 6 and 12 (12), may display intellectual disabilities and/or birth defects, due to a partial monosomy or trisomy. In addition, Robertsonian translocations, such as the rather common translocation $14 / 21$, can result in offspring with Down syndrome or in gestational loss of a conceptus with monosomy of chromosome 14 or 21, or trisomy of chromosome 14, which are not compatible with life.

Assisted reproductive techniques have given the chance of having a child to infertile males with azoospermia and oligozoospermia. However, using the intracytoplasmic sperm injection (ICSI) in this group with high genetic abnormality ratio may increase the inheritance of paternal genetic disorders to offspring (13). It occurs because the presence of the structural chromosomal abnormality predisposes to abnormal segregation in meiosis. Alternate segregation in male gametes has been reported and may result in in vitro fertilization IVF/ICSI failure (14). This may lead to fertilization failure or poor embryonic development after fertilization. Significant heterogeneity was observed in the rates of unbalanced gametes, varying from $2.7 \%$ to $26.5 \%$ according to the translocations (15). In these cases, sperm retrieval for IVF/ICSI procedures should be indicated with restrictions, and the couple should be offered preimplantation diagnosis whenever possible or sperm donation.

Polymorphic variants, particularly involving the heterochromatic region of chromosomes 1, 9, 16, $\mathrm{Y}$ and the nucleolar organizing region of acrocentric chromosomes, are known to occur in $2.7 \%$ of the general population (16). However, much higher frequencies $(12.2-38 \%)$ have been reported in infertile individuals $(17,18)$. In the present study, the overall occurrence of chromosomal variants was $8.3 \%$ and included enlarged heterochromatin on the long arm of chromosome $9(9 \mathrm{qh}+)$, pericentromeric inversion of chromosome $9(9 \mathrm{ph})$, and enlarged satellites of acrocentric chromosomes $\left(14 \mathrm{ps}^{+}, 15 \mathrm{ps}^{+}, 21 \mathrm{ps}^{+}\right.$, and $22 \mathrm{ps}+$ ). Inversions (such as $9 \mathrm{ph}$ ) can disturb spermatogenesis and lead to the production of unbalanced gametes through the formation of an inversion loop, even if the inverted region includes only heterochromatin (19). Enlarged heterochromatin $(9 \mathrm{qh}+)$ could make synapse difficult and, as a consequence, may delay or prevent it. One patient who presented oligoasthenozoospermia had both the 9ph and the $9 \mathrm{qh}+$ variants. This case was reported previously (20), and we believe that the morphological difference between the homologous chromosomes 9 could have led to an error in crossing-over and, as a consequence, produced aberrant gametes. Chromosomal variations associated with male infertility, including structural or numerical chromosomal abnormalities 
and quantitative or positional modifications of the constitutive heterochromatin, have been shown to affect male gamete formation and function possibly due to the silencing effect of these heterochromatic variations on otherwise normally expressed genes (21). We can, however, not rule out the possibility that the concomitant finding of azoospermia and chromosomal polymorphic variants in two patients (46,XY,14ps+ and 46,XY,21ps+, Table-3) was coincidental.

Deletions in the AZFa region usually lead to Sertoli-cell-only syndrome (SCOS). The AZFb region is involved in the regulation of meiosis (22). Complete deletions of $\mathrm{AZFb}$ or $\mathrm{AZFb}+\mathrm{c}$ lead to azoospermia associated with SCOS or pre-meiotic spermatogenic arrest. AZFc deletions lead to azoospermia or severe oligozoospermia, associated with different spermatogenic phenotypes in the testis (1). In the present study, $4.2 \%(6 / 143)$ of the infertile patients had Y-chromosome microdeletions, while in previous studies on infertile men, Hassum-Filho et al. (2005) (23) found 3.45\% (1/29) of Y-chromosome microdeletions, and Pieri et al. (2006) (24) found $8.8 \%(10 / 114)$ of microdeletions. In the present study, the protocol used to investigate Y-chromosome microdeletions includes one gene of each AZF region and can detect the three major deletions on the Y-chromosome related to idiopathic spermatogenic failure. In the study of Hassum-Filho et al. (2005) (23), 18 regions were investigated, while Pieri et al. (2006) (24) started their analysis with 19 regions and then decided to investigate the six most frequent ones. The number of investigated genes may not influence the results of the deletion and prognosis, but may allow a better characterization of the extent of the deletion. In the literature, the frequency of Ychromosome microdeletions ranges from 0 to $60 \%$, according to patient selection and Y-chromosome regions studied (25-27).

The phenotypes associated with microdeletions in the different AZF regions are variable $(26,28)$. As there is no precise association between genotype and testicular histology, no well established standard treatment or prognostic criterion exists to date for the different chromosome anomalies found in infertile men. Testicular sperm extraction and ICSI for the treatment of male-factor infertility have permitted the use of sperm from oligozoospermic patients with $\mathrm{AZFb}$ and AZFc microdeletions, to achieve successful fertilization and pregnancies (29). For AZFa microdeletions the prognosis is poor, as in these cases there are no germ cells in the testis $(30,31)$.

It has to be considered, however, that testicular sperm retrieval procedures performed on men with severe male infertility of genetic origin might bear a potential risk of transmitting genetic abnormalities to the offspring $(7,28,32)$. If a man with an AZFc microdeletion takes part in an ICSI procedure, he can pass the same deletion, or a larger one, on to his male offspring, since deletions can generate chromosomal instability and lead to deletions of the nearby regions, in further generations (33). In view of the genetic risk for the subsequent generations, the importance of a careful evaluation of karyotypes and AZF microdeletions in men presenting idiopathic infertility with non-obstructive azoospermia or severe oligozoospermia, prior to assisted reproduction by ICSI, is evident.

In conclusion, our results support the existence of a relationship between genetic abnormalities such as chromosomal aberrations/variants and Y-chromosome microdeletions and non-obstructive azoospermia and severe oligozoospermia. The high frequency of genetic alterations $(18.8 \%)$ found in the men affected by idiopathic infertility with severe oligozoospermia and non-obstructive azoospermia strongly suggests that such patients should be karyotyped and receive counseling before they are referred for assisted reproduction techniques. Moreover, these investigations can minimize the risk of transmitting genetic abnormalities to future generations, such as genetic male infertility, intellectual disability, genital ambiguity and/or birth defects.

\section{ACKNOWLEDGEMENT}

Supported by Center for Study of Genetics and Human Reproduction, ABC Medical School.

\section{CONFLICT OF INTEREST}

None declared. 


\section{REFERENCES}

1. Ferlin A, Arredi B, Foresta C: Genetic causes of male infertility. Reprod Toxicol. 2006; 22: 133-41.

2. Shefi S, Turek PJ: Definition and current evaluation of subfertile men. Int Braz J Urol. 2006; 32: 385-97.

3. Krausz C, Degl'Innocenti S: Y chromosome and male infertility: update, 2006. Front Biosci. 2006; 11: 304961.

4. Foresta C, Ferlin A, Gianaroli L, Dallapiccola B: Guidelines for the appropriate use of genetic tests in infertile couples. Eur J Hum Genet. 2002; 10: 303-12.

5. World Health Organization: WHO laboratory manual for the examination of human semen and semencervical mucus interaction. Cambridge: Cambridge University Press, 1999. 4th ed.

6. Shaffer LG, Tommerup N: ISCN 2005 - An international system for human cytogenetic nomenclature. Basel: S. Karger; 2005; pp 37-84.

7. Pinkel D, Landegent J, Collins C, Fuscoe J, Segraves R, Lucas J, et al.: Fluorescence in situ hybridization with human chromosome-specific libraries: detection of trisomy 21 and translocations of chromosome 4. Proc Natl Acad Sci U S A. 1988; 85: 9138-42.

8. Umeno M, Shinka T, Sato Y, Yang XJ, Baba Y, Iwamoto $\mathrm{T}$, et al.: A rapid and simple system of detecting deletions on the $\mathrm{Y}$ chromosome related with male infertility using multiplex PCR. J Med Invest. 2006; 53: 147-52.

9. Pina-Neto JM, Carrara RC, Bisinella R, Mazzucatto LF, Martins MD, Sartoratto E, et al.: Somatic cytogenetic and azoospermia factor gene microdeletion studies in infertile men. Braz J Med Biol Res. 2006; 39: 555-61.

10. Rajender S, Rajani V, Gupta NJ, Chakravarty B, Singh L, Thangaraj K: SRY-negative 46,XX male with normal genitals, complete masculinization and infertility. Mol Hum Reprod. 2006; 12: 341-6.

11. Van Assche E, Bonduelle M, Tournaye H, Joris H, Verheyen G, Devroey P, et al.: Cytogenetics of infertile men. Hum Reprod. 1996; 11 (Suppl 4): 1-24; discussion 25-6.

12. Bianco B, Christofolini D, Gava M, Mafra F, Moraes E, Barbosa C. Severe oligospermia associated with a unique balanced reciprocal translocation $\mathrm{t}(6 ; 12)(\mathrm{q} 23 ; \mathrm{q} 24.3)$ : male infertility related to $\mathrm{t}(6 ; 12)$. Andrologia. 2011;43:145-8.

13. Kosar PA, Ozçelik N, Kosar A: Cytogenetic abnormalities detected in patients with non-obstructive azoospermia and severe oligozoospermia. J Assist Reprod Genet. 2010; 27: 17-21.
14. Scriven PN, Flinter FA, Braude PR, Ogilvie CM: Robertsonian translocations--reproductive risks and indications for preimplantation genetic diagnosis. Hum Reprod. 2001; 16: 2267-73.

15. Moradkhani K, Puechberty J, Bhatt S, Lespinasse J, Vago P, Lefort G, et al.: Rare Robertsonian translocations and meiotic behaviour: sperm FISH analysis of $\mathrm{t}(13 ; 15)$ and $\mathrm{t}(14 ; 15)$ translocations: a case report. Hum Reprod. 2006; 21: 3193-8.

16. Yakin K, Balaban B, Urman B: Is there a possible correlation between chromosomal variants and spermatogenesis? Int J Urol. 2005; 12: 984-9.

17. Lissitsina J, Mikelsaar R, Punab M: Cytogenetic analyses in infertile men. Arch Androl. 2006; 52: 91-5.

18. Madon PF, Athalye AS, Parikh FR: Polymorphic variants on chromosomes probably play a significant role in infertility. Reprod Biomed Online. 2005; 11: 726-32.

19. Morel F, Laudier B, Guérif F, Couet ML, Royère $\mathrm{D}$, Roux C, et al.: Meiotic segregation analysis in spermatozoa of pericentric inversion carriers using fluorescence in-situ hybridization. Hum Reprod. 2007; 22: 136-41.

20. Belangero SI, Christofolini DM, Bianco B, Gava MM, Wroclawski ER, Barbosa CP: Male infertility related to an aberrant karyotype, 46,XY,9ph,9qh+. Fertil Steril. 2009; 91: 2732-3.

21. Minocherhomji S, Athalye AS, Madon PF, Kulkarni D, Uttamchandani SA, Parikh FR: A case-control study identifying chromosomal polymorphic variations as forms of epigenetic alterations associated with the infertility phenotype. Fertil Steril. 2009; 92: 88-95.

22. Ferlin A, Moro E, Rossi A, Dallapiccola B, Foresta $\mathrm{C}$ : The human $\mathrm{Y}$ chromosome's azoospermia factor $\mathrm{b}$ $(\mathrm{AZFb})$ region: sequence, structure, and deletion analysis in infertile men. J Med Genet. 2003; 40: 18-24.

23. Hassun Filho PA, Cedenho AP, Lima SB, Ortiz V, Srougi M: Single nucleotide polymorphisms of the heat shock protein 90 gene in varicocele-associated infertility. Int Braz J Urol. 2005; 31: 236-42.

24. Pieri Pde C, Pereira DH, Glina S, Hallak J, McElreavey $\mathrm{K}$, Moreira-Filho CA: A cost-effective screening test for detecting AZF microdeletions on the human $\mathrm{Y}$ chromosome. Genet Test. 2002; 6: 185-94.

25. Simoni M, Bakker E, Krausz C: EAA/EMQN best practice guidelines for molecular diagnosis of $\mathrm{y}$-chromosomal microdeletions. State of the art 2004. Int J Androl. 2004; 27: 240-9.

26. Foresta C, Moro E, Ferlin A: Y chromosome microdeletions and alterations of spermatogenesis. Endocr Rev. 2001; 22: 226-39. 
27. Simoni M, Tüttelmann F, Gromoll J, Nieschlag E: Clinical consequences of microdeletions of the Y chromosome: the extended Münster experience. Reprod Biomed Online. 2008; 16: 289-303.

28. Huang WJ, Yen PH: Genetics of spermatogenic failure. Sex Dev. 2008; 2: 251-9.

29. Gambera L, Governini L, De Leo V, Luddi A, Morgante G, Tallis V, Piomboni P. Successful multiple pregnancy achieved after transfer of frozen embryos obtained via intracytoplasmic sperm injection with testicular sperm from an AZFc-deleted man. Fertil Steril. 2010; 94: 2330.e1-3.

30. Krausz C, Quintana-Murci L, McElreavey K: Prognostic value of $Y$ deletion analysis: what is the clinical

\section{Correspondence address:}

Dr. Bianca Bianco

Faculdade de Medicina do ABC

Av. Príncipe de Gales, 821

Prédio CEPES / $2^{\circ}$ andar

Santo André, SP, 09060-650, Brazil

Fax: + 5511 4438-7299

E-mail: bianca.bianco@hotmail.com prognostic value of $\mathrm{Y}$ chromosome microdeletion analysis? Hum Reprod. 2000; 15: 1431-4.

31. Foresta C, Moro E, Ferlin A: Y chromosome microdeletions and alterations of spermatogenesis. Endocr Rev. 2001; 22: 226-39.

32. Cinar C, Yazici C, Ergünsu S, Beyazyürek C, Javadova D, Saglam Y, et al.: Genetic diagnosis in infertile men with numerical and constitutional sperm abnormalities. Genet Test. 2008; 12: 195-202.

33. Lee SH, Ahn SY, Lee KW, Kwack K, Jun HS, Cha KY: Intracytoplasmic sperm injection may lead to vertical transmission, expansion, and de novo occurrence of Y-chromosome microdeletions in male fetuses. Fertil Steril. 2006; 85: 1512-5.

Accepted after revision: September 2, 2010

\section{EDITORIAL COMMENT}

The authors should be congratulated on reporting the incidence of genetic-related infertility in a subset of Brazilian infertile patients presenting with severe oligozoospermia or non-obstructive azoospermia. Clinically available molecular testing may reveal microdeletions in the long arm of the $\mathrm{Y}$ chromosome. This novel molecular diagnosis application reclassified about $10 \%$ of the infertile male population previously misdiagnosed as idiopathic oligozoospermia or non-obstructive azoospermia. The authors highlighted the importance of screening men with non-obstructive azoospermia and severe oligozoospermia for Y-chromosome infertility seeking infertility treatment, and reported that about $5 \%$ of their patient population harbored Yq micro- deletions. Originally, 3 AZF regions were defined: $\mathrm{AZFa}, \mathrm{AZFb}$, and AZFc (azoospermia factors a, b, and $\mathrm{c}$ ), which map on the long arm (Yq) in order from the centromere to the telomere, but $\mathrm{AZFb}$ and AZFc overlaps (1). A fourth region, named AZFd, located between $\mathrm{AZFb}$ and AZFc was also reported. The relative frequency of individual microdeletions are $60 \%, 5 \%$ and $16 \%$ for AZFc, AZFa and AZFb regions, but combined deletions occur in about $15 \%$ of the cases (2). Because the deletions tend to occur between large palindromic repeats, Repping et al. (2002) proposed a more appropriate nomenclature using the name of the flanking repeats for the types of recurrent deletions (1). Not all men presenting with AZF microdeletions and azoospermia are 
sterile. Sperm can be found in the testis with $70 \%$ of the cases in partial and complete AZFc deletion azoospermic patients. In contrast, the chance of finding sperm in azoospermic men with complete AZFa or AZFb deletions is unlikely (3). It seems that the presence of a deletion of the Yq chromosome has no apparent negative effect on fertilization or pregnancy rates and it does not increase the risk for birth defects in children conceived via assisted reproduction (3). Y chromosome infertility is inherited in a Y-linked manner. Because males with deletion of the AZF regions of the long arm of the $\mathrm{Y}$ chromosome are infertile, the deletions are usually de novo and therefore not present in the father of the proband. However, the male offspring have the same deletion as their father, with a high risk of male infertility. The authors have highlighted the importance of adequate counseling for this group of patients, and added to the discussion since deletions can generate chromosomal instability and lead to deletions of the nearby regions.

It was also interesting to note that about $7 \%$ of their oligozoospermic population presented with chromosomal abnormalities, which can be easily detected by karyotyping. Due to the low cost, availability and clinical implications of genetic testing for azoospermic and severe oligozoospermic males, it now clear that such screening should be offered to males who meet these criteria and are seeking infertility treatment.

\section{REFERENCES}

1. Repping S, Skaletsky H, Lange J, Silber S, Van Der Veen F, Oates RD, et al.: Recombination between palindromes P5 and P1 on the human Y chromosome causes massive deletions and spermatogenic failure. Am J Hum Genet. 2002; 71: 906-22.

2. Shefi S, Turek PJ: Definition and current evaluation of subfertile men. Int Braz J Urol. 2006; 32: 385-97.

3. Hopps CV, Mielnik A, Goldstein M, Palermo GD, Rosenwaks Z, Schlegel PN: Detection of sperm in men with $Y$ chromosome microdeletions of the $\mathrm{AZFa}, \mathrm{AZFb}$ and AZFc regions. Hum Reprod. 2003; 18: 1660-5.

Dr. Sandro Esteves

ANDROFERT, Center for Male Reproduction

Campinas, Sao Paulo, Brazil

Fax: + 5519 3295-8877

E-mail:s.esteves@androfert.com.br 